\title{
DDX5-targeting fully human monoclonal autoantibody inhibits proliferation and promotes differentiation of acute promyelocytic leukemia cells by increasing ROS production
}

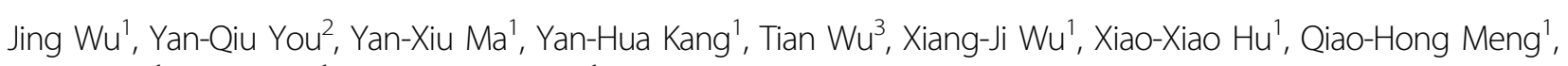
Yin Huang ${ }^{1}$, Na Zhang ${ }^{1}$ and Xiao-Ben Pan ${ }^{1}$

\begin{abstract}
Acute promyelocytic leukemia (APL) therapy involves the compounds cytotoxic to both malignant tumor and normal cells. Relapsed APL is resistant to subsequent chemotherapy. Novel agents are in need to kill APL cells selectively with minimal toxicity. DDX5 has been recognized to be a novel target to suppress acute myeloid leukemia (AML). However, the role of DDX5 remains elusive in APL. Here a DDX5-targeting fully human monoclonal autoantibody named after 2F5 was prepared. It is demonstrated that 2F5 selectively inhibited APL cell proliferation without toxicity to normal neutrophil and tissues. Moreover, 2F5 was confirmed to induce G0/G1 phase arrest in APL cells, and promote APL cell differentiation combined with decreased DDX5 expression and increased reactive oxygen species (ROS) production. Knockdown of DDX5 by siRNA also inhibited proliferation, promoted cell differentiation and enhanced ROS production in APL cells. However, the ROS inhibitor reversed the effects of 2F5 on DDX5 and ROS in APL cells. Thus, we conclude that DDX5-targeting 2F5 inhibits APL cell proliferation, and promotes cell differentiation via induction of ROS.

$2 \mathrm{~F} 5$ showed the therapeutic value of fully human monoclonal autoantibody in APL, which provides a novel and valid approach for treatment of relapse/refractory APL.
\end{abstract}

\section{Introduction}

Acute promyelocytic leukemia (APL) is a subtype of acute myeloid leukemia (AML) characterized by specific biological and clinical features. APL is distinguished by $\mathrm{t}$ $(15 ; 17)$ chromosomal translocation ${ }^{1}$, which causes the production of a fusion protein known as promyelocytic

\footnotetext{
Correspondence: Na Zhang (zhangna@hznu.edu.cn) or

Xiao-Ben Pan (panxiaoben@hznu.edu.cn)

${ }^{1}$ Department of Basic Medicine of Medical School, Department of Infectious Diseases of Affiliated Hospital, Institute of Liver and Metabolic Diseases, Key Laboratory of Aging and Cancer Biology of Zhejiang Province, and Key Laboratory of Inflammation and Immunoregulation of Hangzhou, Hangzhou Normal University, 310000 Hangzhou, Zhejiang, P.R. China

${ }^{2}$ Department of Laboratory Medicine, The Second Affiliated Hospital of Harbin Medical University, 150001 Harbin, Heilongjiang, P.R. China

Full list of author information is available at the end of the article These authors contributed equally: Jing Wu, Yan-Qiu You, Yan-Xiu Ma Edited by M. Diederich
}

leukemia-retinoic acid receptor (PML-RAR $\alpha)^{2}$. APL has been characterized by early onset of clinical signs, disseminated intravascular coagulation and poor response to chemotherapy. Though previously marked by high mortality, it is nowadays the most curable form of $\mathrm{AML}^{3}$. AML therapy is comprised of therapeutic agents that induce apoptosis or promote the differentiation of cancer cells. At present, APL is treated by all-trans retinoic acid (ATRA) in combination with arsenic trioxide (ATO) or by ATRA and chemotherapy ${ }^{4-6}$. However, the resistant to ATRA and ATO of relapse/refractory APL patients is recognized as a critical problem in clinical practice ${ }^{7}$. Therefore, finding alternative targeting drugs with low toxicity may bring prospective solution to the treatment of relapse/refractory APL. 
It has been demonstrated that AML patients had a complex karyotype which is marked by aberration expression of dead-box helicases ${ }^{8}$. Dead-box helicase 5 (DDX5) is a member of this family. Experimental depletion of DDX5 inhibits proliferation of AML cells and induces apoptosis by promoting the production of ROS ${ }^{9}$. Similarly, DDX5 is required in T-cell acute lymphoblastic leukemia (T-ALL) pathogenesis, which is evidenced by the decreased survival rate and inhibited proliferation following depletion of DDX $5^{10}$. All these findings indicated that DDX5 may be a potential drug target in the treatment of APL.

Herein, a DDX5-targeting fully human monoclonal autoantibody named after $2 \mathrm{~F} 5$ was prepared. And then the application potential of 2F5 in the therapy of APL was assessed. Results showed that 2F5 not only markedly inhibited the proliferation of APL cells, but also promoted APL cell differentiation by increasing ROS production. Considering the nontoxicity of $2 \mathrm{~F} 5$ in cell viability, this study could provide a basis for the potential use of $2 \mathrm{~F} 5$ in relapse/refractory APL therapy.

\section{Materials and methods}

\section{Ethics statement}

Experiments involving human and animal samples were approved by the Research Ethics Review Committee of Hangzhou Normal University. Animal procedures performed in this work followed guidelines in accordance with the Regulations for the Administration of Affairs Concerning Experimental Animals. Written informed consents were obtained from all participants.

\section{The preparation of DDX5-targeting fully human monoclonal autoantibody}

Monoclonal antibodies were generated with hybridoma technology. SPYMEG (MBL, Nagoya, Japan) ${ }^{11,12}$ was used as a fusion partner cell for generating human monoclonal antibody that recognizes DDX5 specifically. Peripheral blood mononuclear cells (PBMCs) were obtained from the blood sample of SLE patient, and then were fused with SPYMEG to yield hybridomas. The resulting hybridomas were screened for DDX5-specific antibody secretion and cloned by limiting dilution. One stable clone secreting anti-DDX5 human monoclonal autoantibody was obtained and named after 2F5. The specific binding and affinity between 2F5 and DDX5 (OriGene, Rockville, USA) was determined by Surface Plasmon Resonance (Biacore X100, GE, USA) (Fig. S1b).

\section{Cell lines and culture}

The human APL cell lines (HL-60 and NB4), T-ALL cell lines (Jurkat and CEM-C7), and monocytic leukemia cell line (THP-1) were purchased from Jennio Biotechnology Co., Ltd (Guangzhou, Guangdong, CHN). Blood samples were obtained from healthy volunteer. Neutrophils were isolated with human neutrophil isolation Kit (STEMCELL, CA, USA). PBMCs and monocytes were extracted with isolation kit (Solarbio, Beijing, China). Cells were cultured in RPMI 1640 medium supplemented with 10\% fetal bovine serum (Gibco, Waltham, MA, USA) at $37^{\circ} \mathrm{C}$ in a humidified incubator with $5 \% \mathrm{CO}_{2}$. Cells were cultured in culture medium (normal control), and were treated with $2 \mathrm{~F} 5$ or IgG (negative control) with different concentrations $(20,40$, and $80 \mu \mathrm{M})$ for $4,8,12$, and 16 days. Every 4 days, the cultures were established by centrifugation and then the cell pellets were resuspended in fresh corresponding medium respectively.

\section{Animal treatment and pathological examination}

The 8-week-old Balb/c male mice were obtained from the Center for Laboratory Animals, Hangzhou Normal University, and were randomly divided into two groups. Mice were treated with $12 \mu \mathrm{g} / \mathrm{kg} 2 \mathrm{~F} 5$ by intravenous injection $(n=6)$. Control mice received the same volume of saline $(n=6)$. Mice were euthanized with inhalation of anesthesia $24 \mathrm{~h}$ after injection. Tissue samples were fixed, dehydrated, embedded in paraffin, and sectioned into 4$\mu \mathrm{m}$ slices for subsequent H\&E staining. Wright-Giesma staining was performed using a staining kit (Solarbio, Beijing, China).

\section{Cell proliferation assay}

Cell Counting Kit-8 (CCK-8, Sigma Aldrich, St. Louis, MO, USA) was used to detect cell proliferation, according to the manufacturer's protocol. Cells were divided into three groups including 2F5 treatment group, IgG control and normal control group, and then $1 \times 10^{4}$ cells were seeded with $100 \mu \mathrm{L}$ different treatment medium per well in 96-well plate. CCK-8 solution was added to each well, and incubated for $4 \mathrm{~h}$ in the incubator. The absorbance was measured at $450 \mathrm{~nm}$ using a microplate reader. The living cell number in each group was calculated by the formula from standard curve.

\section{Western blotting}

Protein lysates $(50 \mu \mathrm{g} / \mathrm{lane})$ were resolved on appropriate SDS-PAGE gels and transferred to PVDF membrane (Millipore, Billerica, MA, USA). The membranes were blocked, incubated with the indicated primary antibodies at $4{ }^{\circ} \mathrm{C}$ overnight, and then with the appropriate horseradish peroxidase-conjugated secondary antibodies. Blots were quantified with Image J (National Institutes of Health, Bethesda, MD, USA). Primary antibodies from Abcam (Cambridge, USA) were used: DDX5 (\#ab126730), cyclin A2 (\#ab181591), CDK4 (\#ab108357), cyclin D1 (\#ab16663), PCNA (\#ab92552). $\beta$-actin (\#AC026, ABclonal, Wuhan, China) was used as an internal control. 


\section{NBT reduction assay}

Cells were harvested in number of $1 \times 10^{6}$ and incubated with a 1:1 mixture of culture medium and PBS containing $2 \mathrm{mg} / \mathrm{mL}$ nitro blue tetrazolium (NBT) and $1 \mu \mathrm{g} / \mathrm{mL}$ phorbol 12-myristate 13-acetate (Sigma Aldrich, St. Louis, MO, USA) at $37^{\circ} \mathrm{C}$ for $1 \mathrm{~h}$. The reaction was stopped by adding $200 \mu \mathrm{L}$ of solution of hydrochloric acid $(1 \mathrm{M})$. The absorbance at $562 \mathrm{~nm}$ was measured by spectrophotometer (Bio-Rad, Hercules, CA, USA).

\section{Flow cytometry}

APL cells were incubated with 2F5 with ladder concentrations and $1 \mathrm{mM}$ ATRA (Sigma Aldrich, St. Louis, MO, USA) for $4,8,12$, or 16 days. For flow cytometry analysis, $5 \times 10^{5}$ cells were harvested and washed with PBS, and labeled for 20 min with PE-conjugated antihuman CD11b or CD14 antibody (BD Biosciences, San Jose, CA). After washing with PBS, cells were analyzed on flow cytometry (BD Biosciences, San Jose, CA).

\section{ROS detection}

The intracellular ROS was determined by ROS assay kit (Solarbio, Beijing, China). In brief, cells in different treatment groups were harvested, rinsed with $\mathrm{PBS}$ and incubated with DCFH-DA at $37^{\circ} \mathrm{C}$ for $30 \mathrm{~min}$. Then the DCF fluorescence of the cells was detected by fluorospectrophotometer (Bio-Rad, Hercules, CA, USA) or flow cytometry at an excitation wavelength of $488 \mathrm{~nm}$ and an emission wavelength of $535 \mathrm{~nm}$. Five micromolar of NAcetyl-L-cysteine (NAC, APExBIO, Houston, USA) was added to culture medium for blocking the production of ROS. As a ROS positive control, cells were treated with $100 \mu \mathrm{M} \mathrm{H}_{2} \mathrm{O}_{2}$ for $48 \mathrm{~h}$ as described previously ${ }^{13}$.

\section{Cell cycle analysis}

Cells $\left(1 \times 10^{6} / \mathrm{mL}\right)$ were harvested and were centrifuged. And then the cells were fixed with cold $70 \%$ ethanol overnight at $4{ }^{\circ} \mathrm{C}$, and then were washed twice by cold PBS. Cells were resuspended with $0.5 \mathrm{~mL}$ PI/RNase staining solution. After gentle vortex, cells were incubated for $30 \mathrm{~min}$ in room temperature, protected against light. Then cell cycle was analyzed by flow cytometry. The cell cycle data was fitted and analyzed by ModFit LT (verity software house, USA).

\section{siRNA transfection}

Small interference RNA (siRNA) targeting DDX5, siDDX5 \#1, 5'-GGUGCAGCAAGUAGCUGCUGAAUAU$3^{\prime}$; siDDX5 \#2, 5'-GGAAUCUUGAUGAGCUGCCUAAAU U-3'; and its negative control scrambled siRNA were synthesized from Sangon Biotech (Shanghai, China) Co., Ltd. siRNA targeting DDX5 and control siRNA were transfected to HL-60 and NB4 cells according to the manufacturer's instructions. Briefly, cells were transfected with $50 \mathrm{nM}$ siRNA in serum-free and antibiotics-free DMEM containing $5 \mu \mathrm{L}$ of Lipofectamine 3000 (Invitrogen, Carlsbad, CA, USA). The medium was changed $6 \mathrm{~h}$ later with normal growth medium supplemented with FBS. After $96 \mathrm{~h}$ of transfection cells were harvested.

\section{Surface plasmon resonance (SPR)}

SPR is used to generate affinity information on specific interactions between 2F5 and DDX5 (Biacore X100, GE, USA). An anti-IgG antibody was firstly covalently immobilized onto a CM5 chip. Then $25 \mu \mathrm{M}$ 2F5 were captured noncovalently onto the surface via their Fc region providing an optimal analyte-binding orientation. The resulting complex was stabilized by crosslinking with EDC/NHS to avoid baseline drift during measurement and regeneration. Finally, the interaction between 2F5 and DDX5 was monitored. PBS was used as running buffer in the binding assay. DDX5 $(100 \mathrm{nM})$ were injected at a flow rate of $50 \mu \mathrm{L} / \mathrm{min}$. After association and dissociation of the analyte, a regeneration step was performed in order to remove the remaining bound analyte.

\section{Statistical analysis}

Data are presented as mean \pm standard error of the mean (SEM), and all the experiments were repeated at least three times. Statistical analysis was performed using SPSS 13.0. One-way analysis of variance (ANOVA) was used to compare the differences between control and treatment groups. $p<0.05$ was considered statistically significant.

\section{Results \\ APL cell proliferation inhibited by monoclonal autoantibody $2 \mathrm{~F} 5$}

The effect of 2F5 on cell proliferation of different leukemia cell lines was detected by CCK- 8 assay. Five cell lines including NB4, HL-60, Jurkat, CEM-C7, and THP-1 cells were treated with $40 \mu \mathrm{M} 2 \mathrm{~F} 5$, respectively for 4 days. Human neutrophil, PBMC, and monocyte were used as healthy controls. The results of CCK- 8 assay indicated that 2F5 could significantly inhibit cell proliferation of NB4 and HL-60 cells compared with nontargeting IgG and normal control group (Fig. 1a, b, ${ }^{* * * *} p<0.001$ ). However, 2F5 had no effect on cell proliferation of other three leukemia cell lines as well as the healthy controls (Fig. 1c-h). Cyclin D1 and PCNA expression levels in NB4 and HL-60 cells were analyzed for 4 days after 2F5 treatment with three different concentrations $(20,40$, and $80 \mu \mathrm{M})$. Compared with both nontargeting IgG and normal control group, 2F5 induced a significant dosedependent decrease of cyclin D1 and PCNA expression levels in NB4 and HL-60 cells (Fig. S2). 


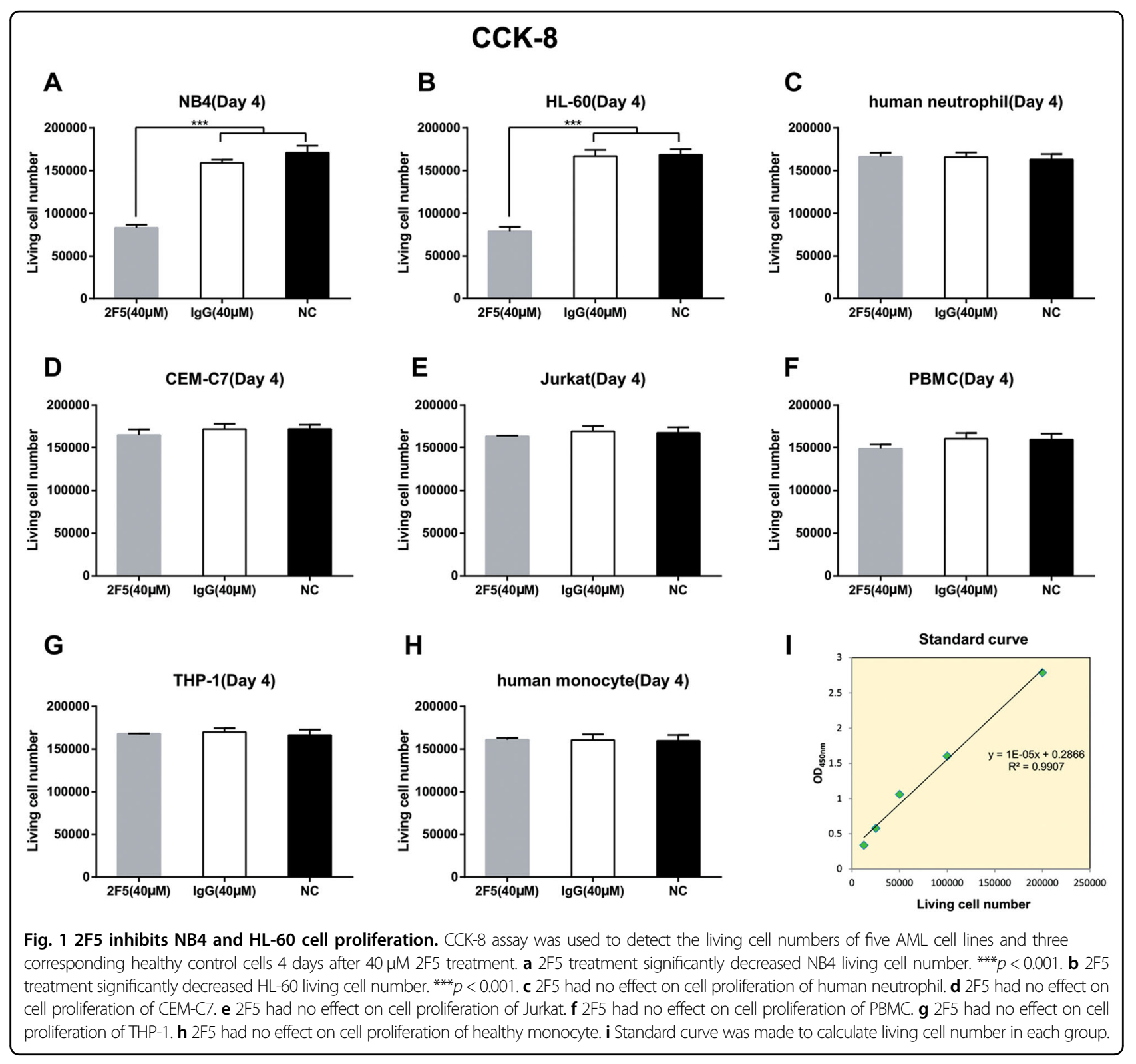

\section{Expression of DDX5 was elevated in APL cell lines}

The expression of DDX5 was detected in five different leukemia cell lines, and corresponding healthy control cells. As shown in Fig. 2, DDX5 expression levels in NB4 and HL-60 cells were much higher than the other six cell lines ( $* p<0.05$, Fig. 2a, b). Additionally, DDX5 levels in normal neutrophils, PBMCs and in monocytes were significantly lower than in APL (NB4 and HL-60), T-ALL (Jurkat and CEM-C7), and in monocyte leukemia (THP-1) cell lines $\left({ }^{*} p<0.05,{ }^{* * *} p<0.01\right.$, Fig. 2b).

\section{Downregulated DDX5 expression induced by 2F5 in APL cells}

Five types of different leukemia cells were treated with 2F5. After 4 days, DDX5 expression was detected by western blotting. Results indicated that DDX5 expression were significantly suppressed in NB4 cells by 2 F5 ( $p<$ 0.05 , Fig. $2 \mathrm{~d}, \mathrm{e})$, as well as in HL-60 cells $(* p<0.05$, Fig. $2 \mathrm{f}-\mathrm{h}$ ). However, there was no significant difference of DDX5 expression in CEM-C7, Jurkat, THP-1, healthy neutrophil, PBMC, and monocyte treated with 2F5, compared with IgG or normal control (Fig. $2 \mathrm{i}-\mathrm{n}$ ).

\section{G0/G1 phase arrest induced by $2 \mathrm{~F} 5$ in APL cells}

The cell cycle distribution was analyzed by flow cytometry and western blotting in NB4 and HL-60 cells at day 4 after $40 \mu \mathrm{M} 2 \mathrm{~F} 5$ treatment. A significant increase in the G0/G1 phase was detected both in NB4 $(* * p<0.01$, Fig. 3a-d) and HL-60 cells $(* * * p<0.001$, Fig. 3h-k). Meanwhile, 2F5 significantly decreased the percentages of 


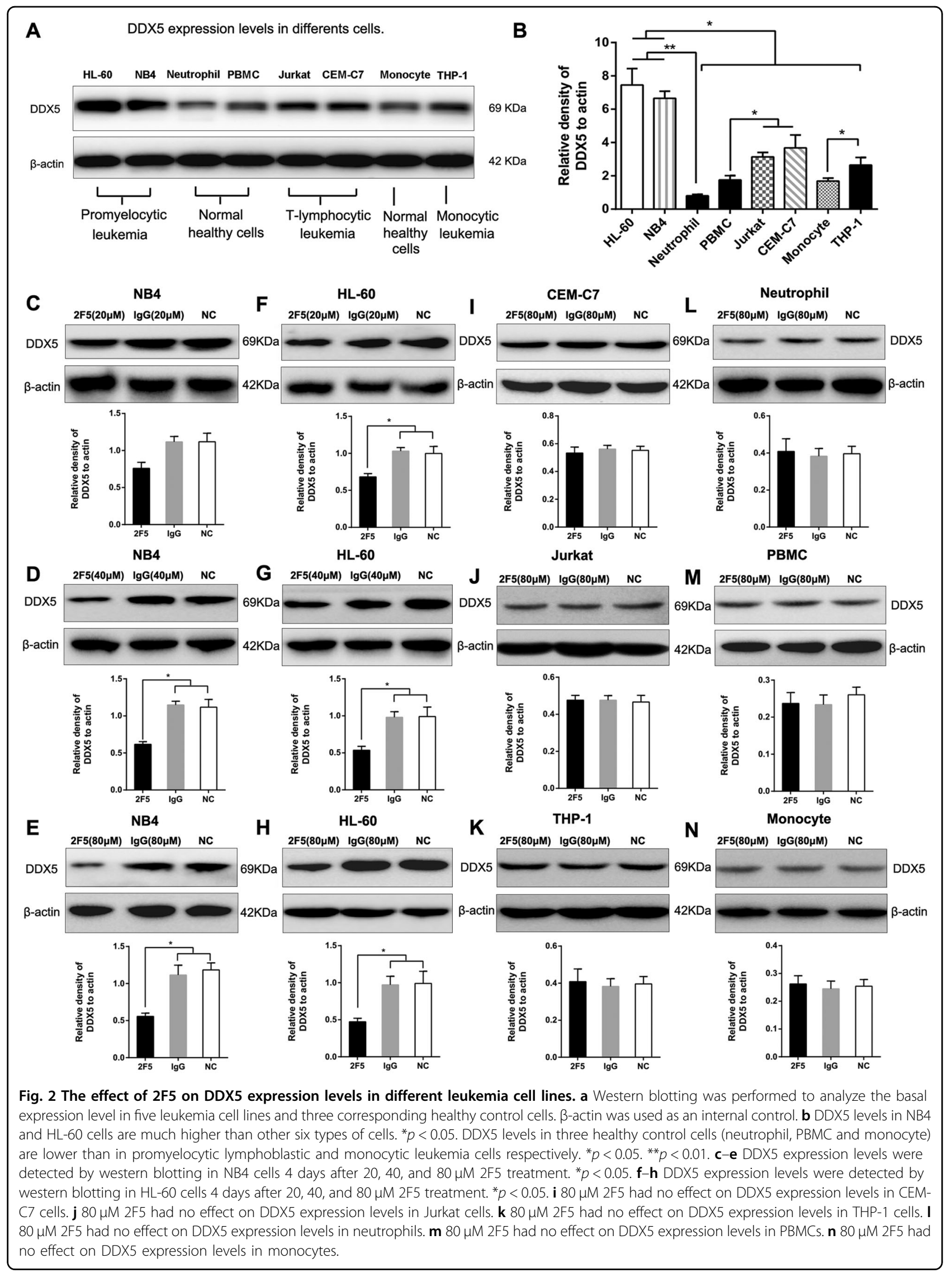




\section{Cell cycle}

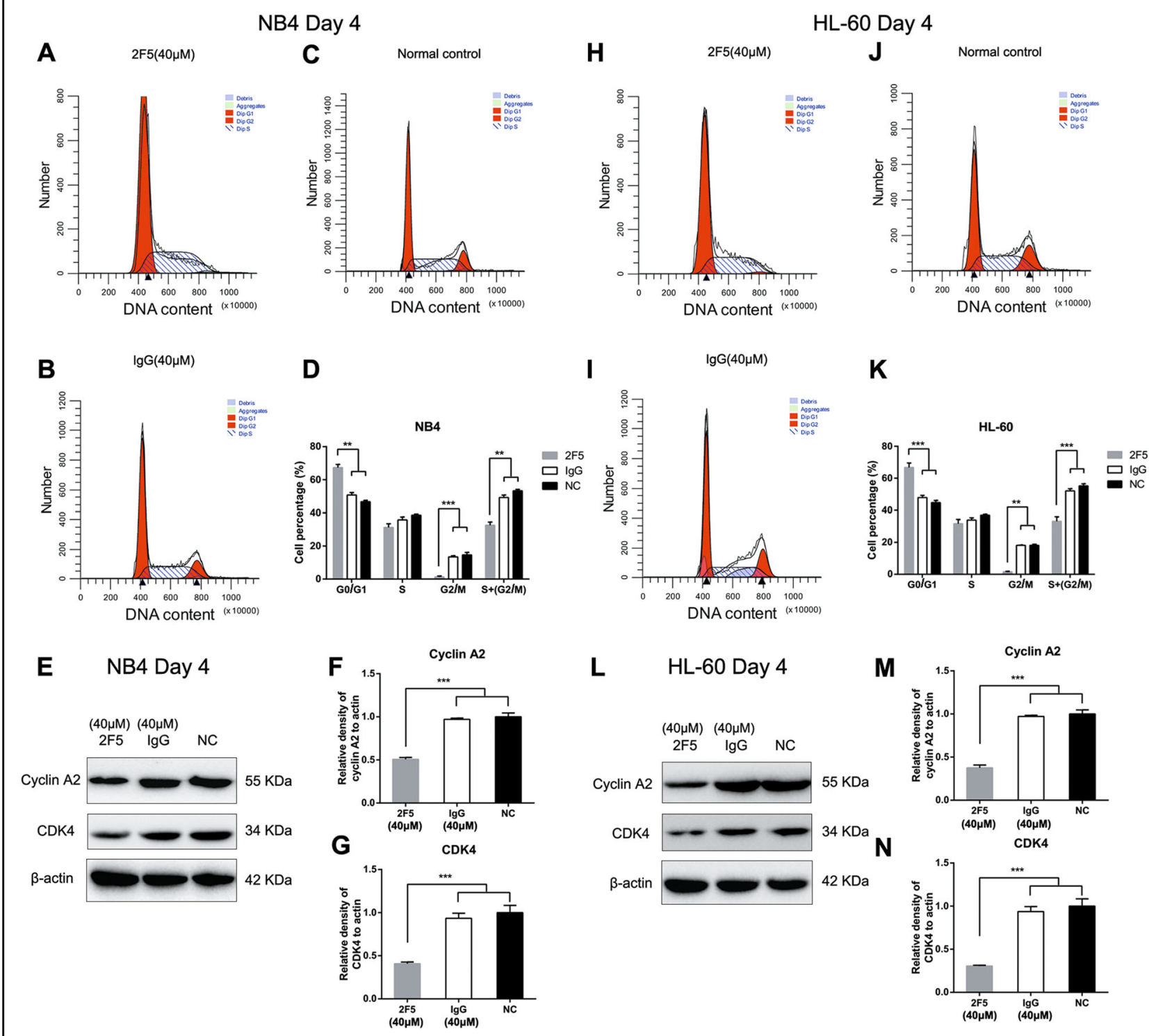

Fig. 32 2F5 induces G0/G1 phase arrest in NB4 and HL-60 cells. a-c Cycle analysis was detected by flow cytometry in NB4 cells treated with $40 \mu M$ $2 F 5,40 \mu \mathrm{M}$ nontargeting $\mathrm{lgG}$, and in untreated NB4 cells. $\mathbf{d}$ Percentage of NB4 cells in G0/G1 phase was higher than lgG and normal control. ${ }^{* * *} p<$ 0.001 . However, percentages of $2 F 5$-treated NB4 cells in G2/M and G2/M plus S phase were lower than lgG and normal control. ${ }^{* *} p<0.01,{ }^{* * *} p<$ 0.001. e- $\mathbf{g}$ Cell cycle related protein cyclin A2 and CDK4 expression levels were detected by western blotting in NB4 cells 4 days after $40 \mu \mathrm{M} 2 \mathrm{~F} 5$ treatment, $40 \mu \mathrm{M}$ non-targeting IgG treatment, and in untreated NB4 cells. 2F5 significantly decreased cyclin A2 and CDK4 expression levels in NB4 cells. ${ }^{* * *} p<0.001$. h-i Flow cytometry was used for cell cycle analysis in $\mathrm{HL}-60$ cells 4 days after $40 \mu \mathrm{M} 2 \mathrm{~F} 5$ treatment, $40 \mu \mathrm{M}$ non-targeting lgG treatment, and in untreated HL-60 cells. $\mathbf{k}$ Percentage of $\mathrm{HL}-60$ cells in G0/G1 phase was higher than IgG and normal control. ${ }^{* *} p<0.01$. However, percentages of 2F5-treated HL-60 cells in G2/M and G2/M plus S phase were lower than IgG and normal control. ${ }^{* *} p<0.01$. ${ }^{* *} p<0.001$. I-n 2F5 significantly decreased cyclin A2 and CDK4 expression levels in HL-60 cells compared with IgG and normal control. ${ }^{* * *} p<0.001$.

G2/M and G2/M plus S phase in NB4 and HL-60 cells $\left({ }^{* * *} p<0.01,{ }^{* * * * *} p<0.001\right.$, Fig. 3d, k). Consistently, cell cycle related protein Cyclin A2 and CDK4 expression levels were also found dramatically decreased in NB4 $\left(^{* * * *} p<\right.$ 0.001 , Fig. $3 \mathrm{e}-\mathrm{g})$ and HL-60 cells $\left({ }^{* * *} p<0.001\right.$, Fig. 3l-n) after 2F5 treatment.
APL cells differentiation induced by $2 \mathrm{~F} 5$ had no harmful effects on normal neutrophils and tissues

To examine whether 2F5 contributed to APL cell differentiation, Wright-Giemsa staining and NBT reduction assay were performed. Morphological features of cell differentiation were observed in 2F5-treated HL-60 cells 
and ATRA positive control group, such as a lower nucleocytoplasmic ratio and chromatin condensation (Fig. S3). Specially, 2F5-treated HL-60 cells showed granulocytic differentiation morphological features, including shrinkage nucleus, lobulated nucleus, and grayish cytoplasm (Fig. S3e, f).

Furthermore, NBT reduction assay was performed to detect cell differentiation. The result of NBT reduction assay showed that in NB4 cells, $40 \mu \mathrm{M} 2 \mathrm{~F} 5$ induce a significant increase of NBT reduction activity at day 4,8 , and 12 after 2F5 treatment $\left(" p<0.05,{ }^{* * *} p<0.01\right.$, ${ }^{* * * *} p<0.001$, Fig. S4a-c). Two curves showed that $2 \mathrm{~F} 5$ played a timedependent increasing effect on NBT reduction in NB4 and HL-60 cells (Fig. S4d, h).

Cells were found dead 8 days after ATRA treatment (Fig. S4 and Fig. 4), suggesting critical toxic effect of ATRA on cells. The population of CD11b-positive and CD14-positive HL-60 cells significantly increased following 2F5 or ATRA treatment compared with control (" $p<$ 0.05 , Fig. $4 \mathrm{~b}, \mathrm{e})$. Interestingly, even if 2F5 exposure was stopped at day 12, the increasing trend of CD11b-positive and CD14-positive cells were kept to day 16. At day 16, CD14-positive HL-60 cells of 2F5 treatment group was higher than that in ATRA positive control group at day 8 (Fig. 4e). The percentage of CD11b-positive and CD14positive cells persistently increased from day 4 to day 16 (Fig. 4c, f). Flow cytometry result showed that 2F5 promoted the cell differentiation with a time-dependent instead of dose-independent manner. In addition, similar results were acquired in NB4 cells that both CD11bpositive and CD14-positive cells were significantly increased by $2 \mathrm{~F} 5$ treatment $(* * * 0<0.001$, Fig. 5).

In order to confirm the biological safety of $2 \mathrm{~F} 5$, neutrophils were extracted from the blood of healthy volunteer. Then cell proliferation was detected by CCK- 8 assay. The results indicated that there was no significant difference of cell proliferation between 2F5-treatment, normal control, and IgG control (Fig. S3m). Morphological staining was also performed on mice tissues. In animal model, no significant pathological changes were found in brain, kidney, or liver of mice with 2F5 intravenous injection (Fig. S3i-k).

\section{ROS production induced by $2 \mathrm{~F} 5$ in NB4 and HL- 60 cells}

It has been reported that increased ROS levels can induce AML cell differentiation ${ }^{14}$, and ROS production is also critical for macrophage differentiation ${ }^{15}$. Hence, we sought to determine ROS level in APL cells treated with $2 \mathrm{~F} 5$ by $\mathrm{CM}-\mathrm{H}_{2}$ DCFDA ROS detection assay. Compared with IgG control, $20 \mu \mathrm{M}$ and $40 \mu \mathrm{M}$ 2F5 induced a significant increase of ROS production at day 4,8 , and $12(* p$ $<0.05, * * p<0.01,{ }^{* * * *} p<0.001$, Fig. $\left.6 \mathrm{a}-\mathrm{h}\right)$. The $80 \mu \mathrm{M}$ 2F5 significantly increased ROS production at day 8 and day $12\left(* * p<0.01,{ }^{* * * * *} p<0.001\right.$, Fig. 6 j-1) except the day 4 compared with IgG control (Fig. 6i). The curve diagraph showed that the effect of $2 \mathrm{~F} 5$ on ROS production in HL60 cells was enhanced with the prolongation of treatment time, but not dose-dependent (Fig. 6d, h, l). These findings were consistent with the results of NBT assay (Fig. S4) and flow cytometry (Fig. 4). Furthermore, increased ROS production was also detected in NB4 cells treated with 2F5, but not in CEM-C7, Jurkat and THP-1 cells (** $p$ $<0.01$, Fig. S5). These results indicated that 2F5 may exert its role to promote leukemia cell differentiation by ROS production.

\section{ROS inhibitor blocked 2F5-induced cell differentiation}

To determine whether ROS are required to maintain the promoting differentiation effect of 2F5, ROS inhibitor NAC was employed in NB4 and HL-60 cells, and then the ROS production, DDX5 expression and cell differentiation were analyzed. Flow cytometric detection of ROS showed that NAC could block the ROS promotion by $2 \mathrm{~F} 5$ in NB4 and HL-60 cells (Fig. 7e, m). Compared with cells treated with $2 \mathrm{~F} 5$ only, ROS production was significantly decreased in APL cells treated with NAC combined 2F5 $(* * * * 0<0.001$, Fig. $7 d, 1)$. Moreover, DDX5 expression was significantly decreased in 2F5-treated NB4 and HL-60 cells, while increased combined with NAC treated group $\left({ }^{* * * *} p<0.001\right.$, Fig. $\left.7 \mathrm{~h}, \mathrm{p}\right)$. These results suggested that the inhibition effect of 2F5 on DDX5 expression could be reversed by ROS inhibitor. In addition, flow cytometry detection of CD14 showed that compared with 2F5treatment group, CD14-positive APL cells treated with 2F5 combined NAC were decreased significantly (Fig. S6). Collectively, these data indicated that the effect of 2F5 on promoting APL cell differentiation and inhibition of DDX5 expression is dependent on ROS production.

\section{The role of DDX5 silence on cell proliferation, ROS production, and cell differentiation}

To determine whether DDX5 plays an essential role in regulating APL cell differentiation, siRNAs targeting DDX5 were transfected in NB4 and HL-60 cells. Western blotting analysis revealed significant decrease of DDX5 expression in siDDX 5 transfected NB4 and HL-60 cells compared to control scramble siRNA respectively ( $p<$ $0.05, * *<<0.01$, Fig. $8 \mathrm{a}-\mathrm{d})$. Compared with siDDX5, 2F5 had similar ability on specific down-regulating DDX5 expression level, which revealed that 2F5 treatment is sufficient to inhibit DDX5 activity. siDDX5 significantly reduced the living cell number of NB4 and HL-60 cells $(* * * 0<001$, Fig. 8e, f). Moreover, similar to 2F5, siDDX 5 also increased ROS production and CD14posistive cell percentages in NB4 and HL-60 cells ${ }^{* * * *} p<$ 0.001, Fig. $8 \mathrm{~m}, \mathrm{~s}, \mathrm{y})$. 


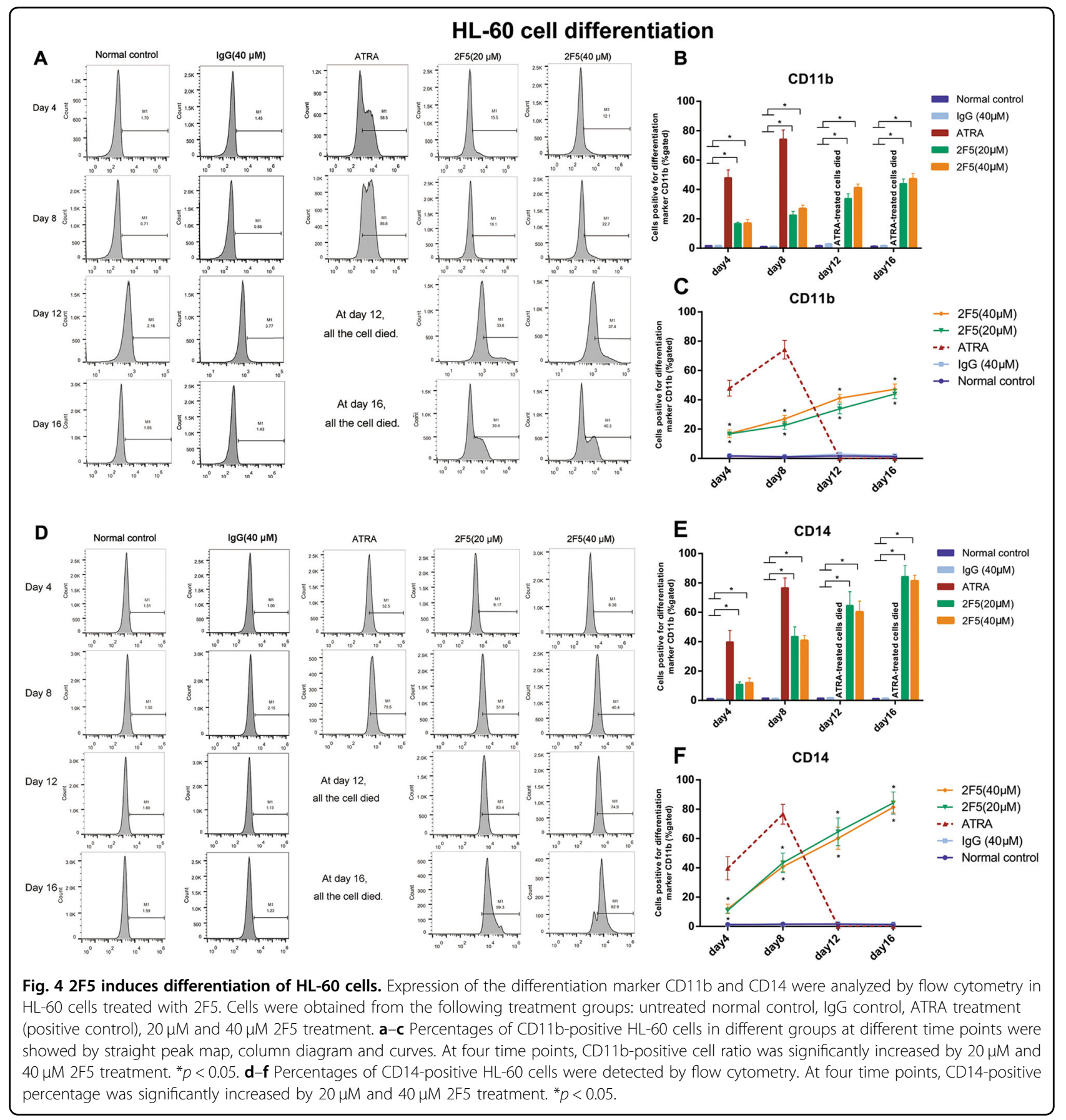

\section{Discussion}

The therapeutic management of APL has considerably evolved during the past two decades. ATRA in combinatorial regimens with chemotherapy has provided high cure rates. But this approach shows significant toxicity including severe myelosuppression and occurrence of relapse/ refractory leukemia ${ }^{16}$. In recent years, the advent of ATO and its use in combination with ATRA with or without chemotherapy has further improved patient outcome by maintaining high antileukemic efficacy. However, common complications such as differentiation syndrome and hepatic toxicity induced by ATO causes serious harm to patients ${ }^{17}$. Monitoring liver function and adjusting treatment regimens at any time during ATO therapy also bring problem and complexity to APL treatment ${ }^{18}$. Therefore, finding new management strategy without toxicity is dramatically valuable for targeting treatment of APL.

Autoantibody is immunoglobulin directed against an individual's self-antigens, the function of which has been abundantly explored in SLE patients. In recent years, 

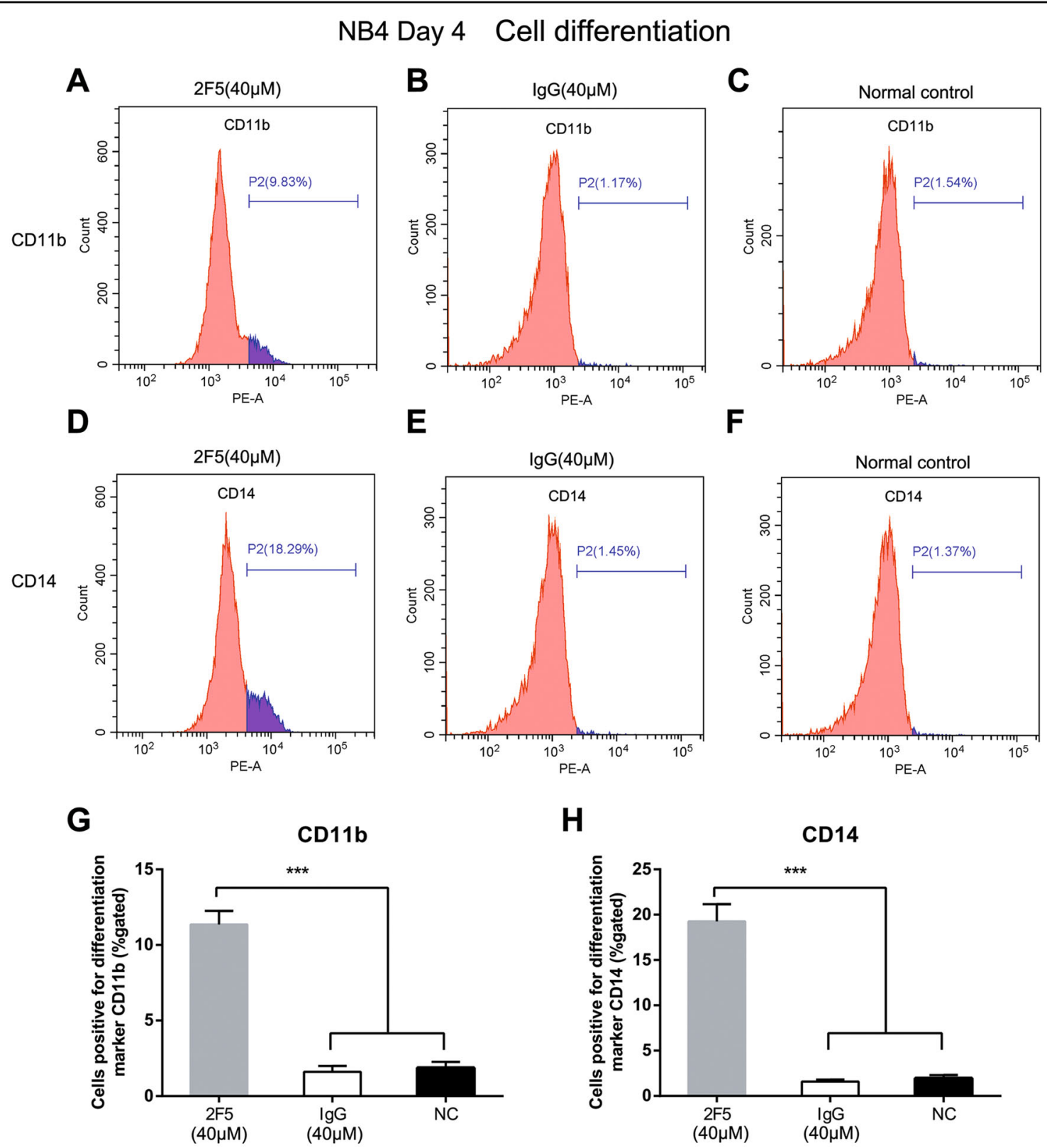

Fig. 52 F5 induces differentiation of NB4 cells. Expression of CD11b and CD14 in NB4 cells were analyzed by flow cytometry 4 days after 2F5 treatment. a-c Percentage of CD11 b-positive NB4 cells in $40 \mu \mathrm{M} 2 \mathrm{~F} 5$ treatment group, $40 \mu \mathrm{M}$ lgG treatment group and in untreated normal group. d-f Percentage of CD14-positive NB4 cells in $40 \mu \mathrm{M} 2 \mathrm{~F} 5$ treatment group, $40 \mu \mathrm{M}$ lgG treatment group and in untreated normal group. $\mathbf{g}$ Percentage of CD11b-positive NB4 cells in 2F5 treatment group was higher than in lgG control and normal control group. ${ }^{* *} p<0.001$. $\mathbf{h}$ Percentage of CD14positive NB4 cells in 2F5 treatment group was higher than in IgG control and normal control group. ${ }^{* *} p<0.001$.

researchers have become more interested in the role and application of autoantibodies in treatment of human malignant tumors ${ }^{19}$. Numerous epidemiological studies showed that risk of some cancers in SLE were decreased ${ }^{20}$. For example, the risk of endometrial cancer in SLE patients is decreased more than $50 \%^{21,22}$, which implies that certain autoantibodies in people with SLE may prevent carcinogenesis.
Currently, existing monoclonal antibody drugs used for treating leukemia mostly targeted lymphocyte surface molecules, such as CD20, CD3, or CD19 and so on ${ }^{23}$. Beside those cell surface biomarkers, some transcriptional regulators have been reported to be aberrantly expressed in many cancers, and are linked to the regulation of many cancer-related pathways. Among those transcriptional regulators, the DDX5 plays a key role in the 


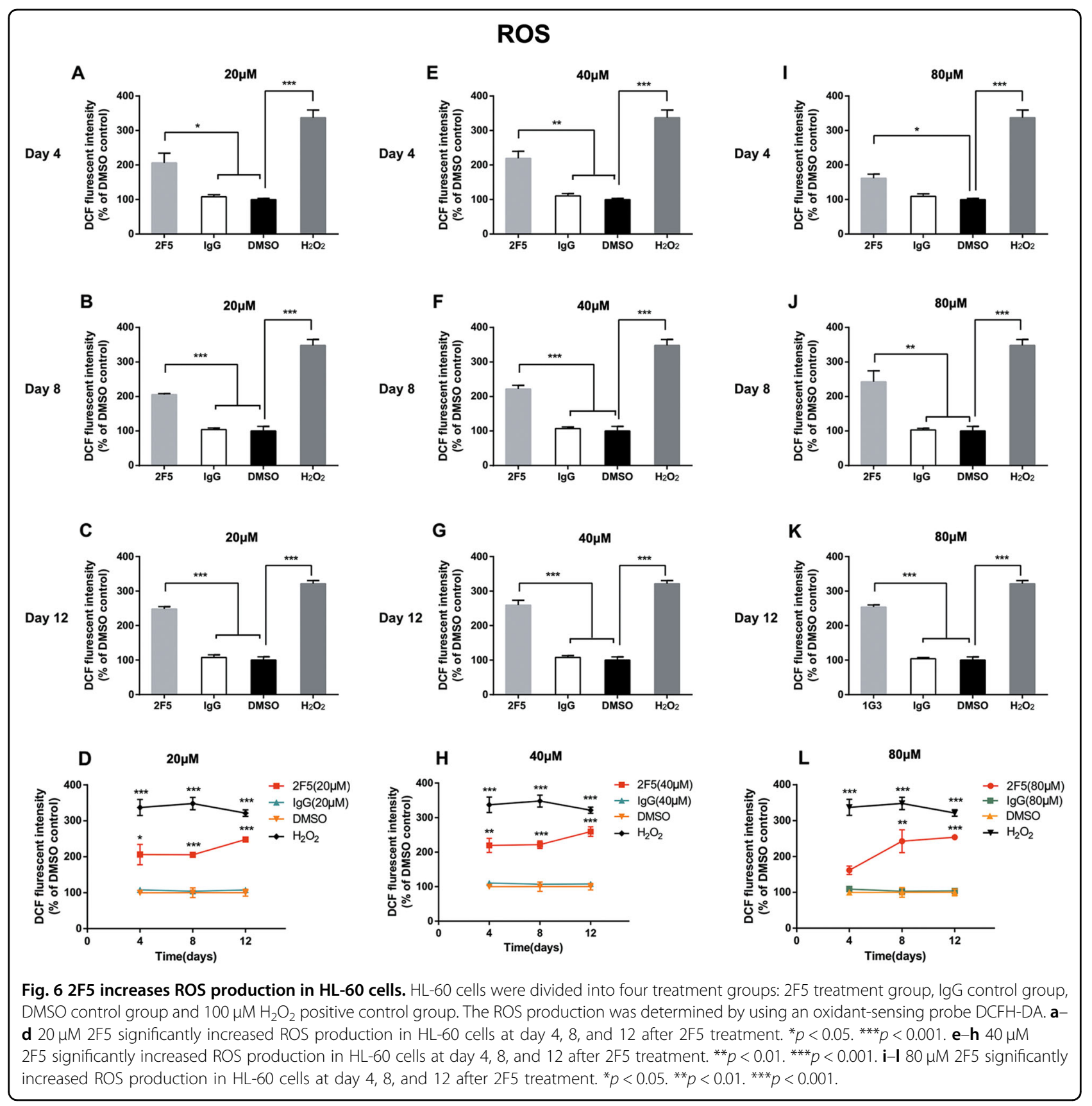

tumorigenesis, invasiveness, and metastasis ${ }^{24}$. It has been reported that AML is dependent on DDX5, and inhibition of DDX5 slows AML cell proliferation in vitro and AML progression in vivo, but is not toxic to cells from normal bone marrow ${ }^{9}$. These investigation conclusions provide an insight that DDX5-targeting monoclonal autoantibodies might be potential new agents for APL therapy.

DDX5 expression is closely correlated with the onset of organ differentiation ${ }^{25}$. The function of DDX5 is responsible for the expression of proteins that contribute to cell differentiation. Otherwise, studies showed that downregulation of DDX5 in gastric and lung cell lines abrogates proliferation $^{26,27}$, which suggested that DDX5 could affect cancer cell proliferation. DDX5 is considered to be required for T-ALL pathogenesis since it regulates the notch signaling pathway which is required for the growth and survival of T-ALL cells. This is evidenced by Lin et al. that DDX5 depletion decreased survival rate and inhibited proliferation of KOPT-K1 and HPB-ALL cells ${ }^{10}$. Similar conclusion was also acquired in present study that both DDX5-targeting 2F5 and siRNA could inhibit APL cell proliferation by downregulation of DDX5. Moreover, 


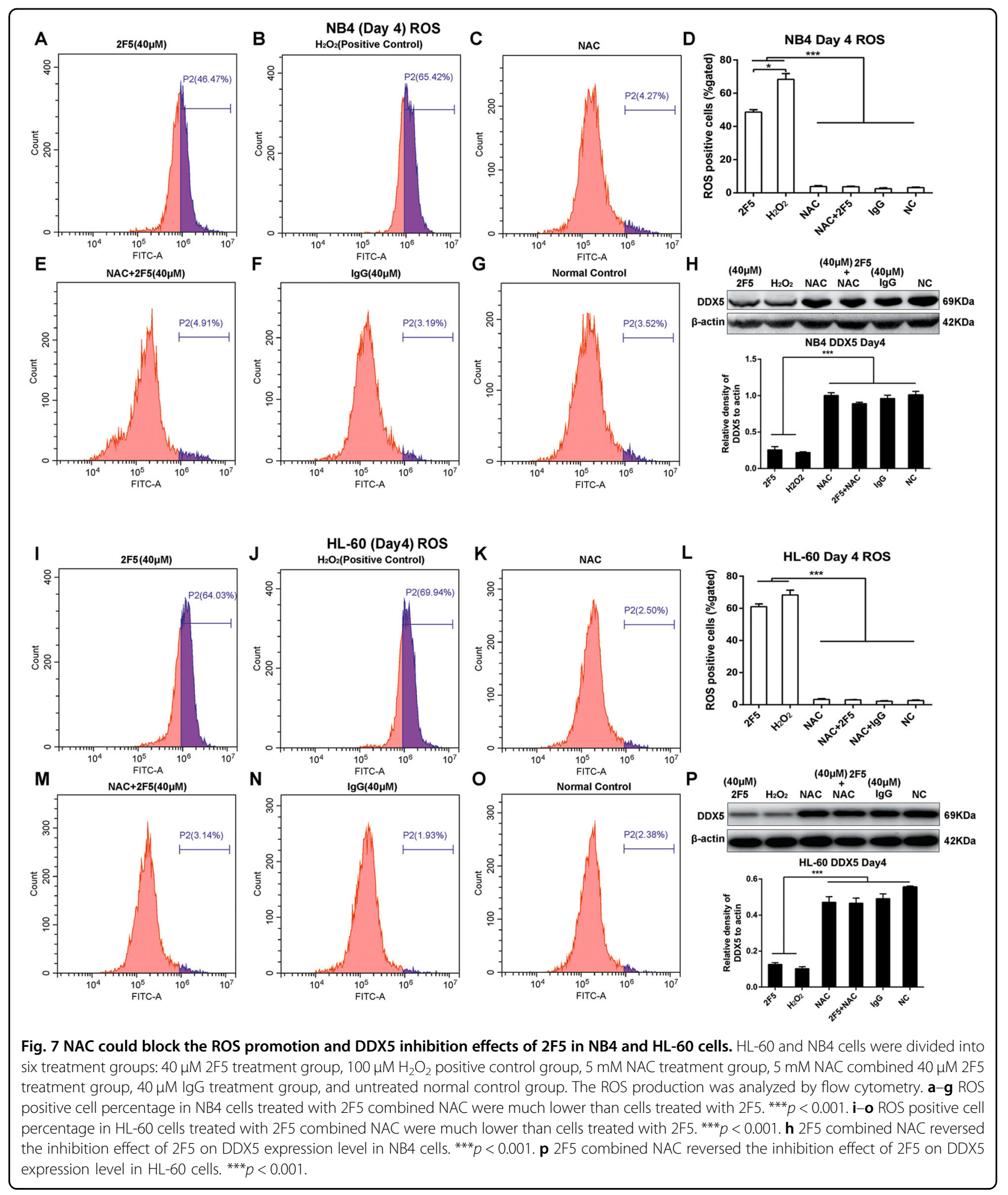

2F5 induced G0/G1 phase arrest in APL cells (Fig. 3). Interestingly, unlike the finding related to DDX5 by Lin et al., we found that DDX5-targeted 2F5 has no effect on the proliferation of T-ALL cell line (Jurkat and CEM-C7).
Our results showed that the basal expression levels of DDX5 in different leukemia cell lines were also different, DDX5 basal level in APL cell lines were much higher than that in T-ALL cell line (Fig. 2). These findings indicated 


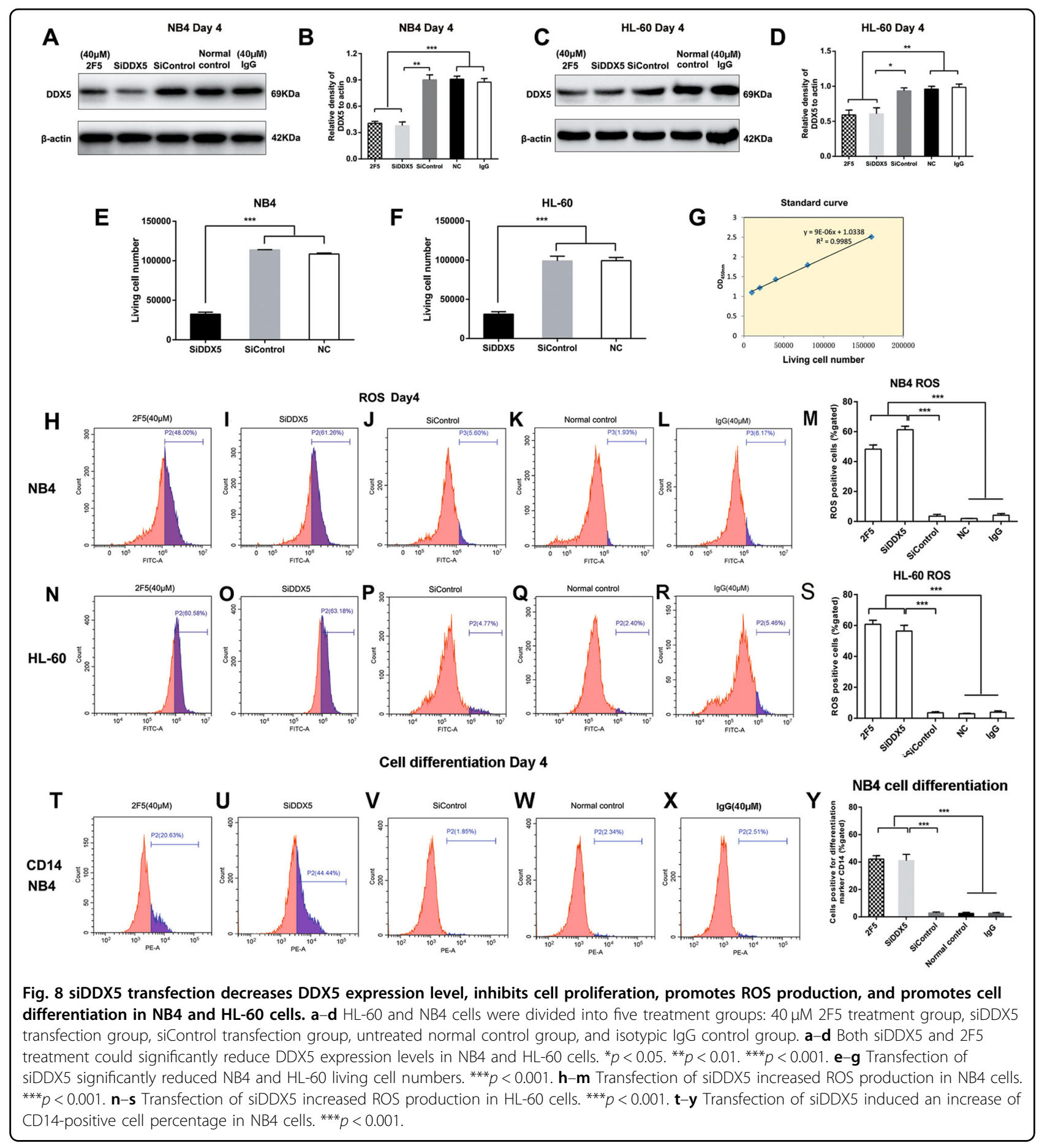

that the different basal level of DDX5 might determine the susceptibility of different leukemia subtypes to $2 \mathrm{~F} 5$. Moreover, 2F5 makes no harmful effect on healthy neutrophils and tissues (Fig. S3). This provides evidence to safety of 2F5 in potential further application.

The differentiation promotion effect of $2 \mathrm{~F} 5$ was evaluated by NBT reduction assay, morphological staining and differentiation biomarker analysis (Figs. 4, 5 and
Figs. S3 and 4). CD11b and CD14 have been known as characteristic surface markers on mature granulocyte and mature monocyte, respectively ${ }^{28}$. Those two surface markers decide the differentiation trends of APL cells. In APL cells treated with $2 \mathrm{~F} 5$, the percentage of CD14positive cells was obviously higher than CD11b-positive cells, thus it is speculated that 2F5 could induce APL cell differentiation along the monocytic lineage more than 
myeloid lineage. Moreover, APL cells were found dead at day 8 after ATRA treatment, while APL cells were found still alive at day 16 after $2 \mathrm{~F} 5$ treatment. It is suggested that 2F5 exerts its pro-differentiation effect on APL cell via other pathway that different from the pro-apoptosis mechanism of $\mathrm{ATRA}^{29}$. Of note, $2 \mathrm{~F} 5$ exerted promotion effect on APL cell differentiation up to day 16, even though the treatment of 2F5 was stopped at day 12 (Fig. 4). It means that $2 \mathrm{~F} 5$ exerts its pro-differentiation effect on APL cells for a long time.

Up to now, there is no monoclonal antibody medicine used clinically for APL therapy with differentiation inducing mechanism. A monoclonal antibody against CD44 used alongside ATRA and FICZ (6-Formylindolo (3, 2-b) carbazole) has been shown to increase cell apoptosis $^{30}$. Another monoclonal antibody called HuM195 is used for APL targeted radiotherapy as a molecular chaperone agent by targeting $\mathrm{CD}_{3} 3^{31}$. HuM195/rGel is a compound prepared by HuM195 coupling to plant toxin gelonin, which has been proven to be cytotoxic to HL-60 cells ${ }^{32}$. As a direct prodifferentiation monoclonal autoantibody, 2F5 has its own specific advantages and mechanism of action. In APL cells, 2F5 was found to inhibit DDX5 expression and promote ROS production significantly. Moreover, siRNAtargeted DDX5 played a similar role to 2F5 on APL cell proliferation, differentiation, and ROS production. However, the ROS inhibitor reversed the effect of $2 F 5$ on DDX5 and ROS in APL cells. These findings indicated that 2F5 could promote APL cell differentiation by inhibition of DDX5 and activation of ROS.

It has been demonstrated that the decrease of DDX 5 is the cause of ROS induction ${ }^{9}$. In addition, it has also been reported that NADPH oxidase-derived ROS plays a critical role in HL-60 cell monocytic differentiation ${ }^{13}$. ROS is involved in the HL-60 cell monocytic differentiation induced by isoliquiritigenin ${ }^{13}$. Combined with results in present study that ROS plays a dual role in promotion effect of APL cell differentiation. On one hand, DDX5 depletion-induced ROS production promotes APL cell differentiation. On the other hand, NAC-mediated ROS inhibition also blocks the decreasing effect of 2F5 on DDX5 and subsequent promotion effect of 2F5 on ROS and cell differentiation (Fig. S7). All these findings suggested that $2 \mathrm{~F} 5$ promoted APL cell differentiation via targeted reducing DDX5-mediated ROS production. For the mechanisms by which autoantibodies penetrate living cells, researchers have suggested that Fc-receptormediated or some special cell membrane proteins mediate endocytosis to facilitate the cell penetration of autoantibodies $^{33}$. However, endosomes prevent 2F5 to recognize and bind to its targeting protein DDX $5^{19}$. For this reason, it is suggested that 2F5 decreased DDX5 level via endosome-independent pathway. It is speculated that
2F5 binds to extracellular nucleotide and transports to the cell plasma via equilibrative nucleoside transporter ${ }^{34}$, and then 2F5 neutralizes free DDX5 consequently (Fig. S7).

This study concluded that DDX5-targeting autoantibody 2 F5 inhibited proliferation, induced a G0/G1 phase arrest, and promoted differentiation of APL cells by decreasing DDX5 and increasing ROS levels without cytotoxicity to healthy tissues and cells. This finding provides a novel and valid approach to solve the problem in therapy of relapse/refractory APL.

\section{Acknowledgements}

This work was supported by grants from the National Natural Science Foundation of China (NSFC) $(81601428 ; 81670530 ; 81700380)$, and the Zhejiang Provincial Natural Science Foundation (ZJNSF) (LY20H250001; LQ19C080001), and the Research Start-Up Funds of Hangzhou Normal University

(2019QDL017; 2018QDL035; 2019QDL008; 2019QDL007).

\section{Author details}

${ }^{1}$ Department of Basic Medicine of Medical School, Department of Infectious Diseases of Affiliated Hospital, Institute of Liver and Metabolic Diseases, Key Laboratory of Aging and Cancer Biology of Zhejiang Province, and Key Laboratory of Inflammation and Immunoregulation of Hangzhou, Hangzhou Normal University, 310000 Hangzhou, Zhejiang, P.R. China. ${ }^{2}$ Department of Laboratory Medicine, The Second Affiliated Hospital of Harbin Medical University, 150001 Harbin, Heilongjiang, P.R. China. PPeking University People's Hospital, Peking University Hepatology Institute, Beijing, P.R. China

\section{Conflict of interest}

The authors declare that they have no conflict of interest.

\section{Publisher's note}

Springer Nature remains neutral with regard to jurisdictional claims in published maps and institutional affiliations.

Supplementary Information accompanies this paper at (https://doi.org/ 10.1038/s41419-020-02759-5).

Received: 13 November 2019 Revised: 7 July 2020 Accepted: 9 July 2020 Published online: 20 July 2020

\footnotetext{
References

1. Ablain, V. et al. Activation of a promyelocytic leukemia-tumor protein 53 axis underlies acute promyelocytic leukemia cure. Nat. Med. 20, 167-174 (2014).

2. Wang, L. et al. Dissection of mechanisms of Chinese medicinal formula Realgar-Indigo naturalis as an effective treatment for promyelocytic leukemia. Proc. Natl Acad. Sci. USA 105, 4826-4831 (2008).

3. Lehmann-Che, J., Bally, C. \& de Thé, H. Resistance to therapy in acute promyelocytic leukemia. N. Engl. J. Med. 371, 1170-1172 (2014).

4. Breccia, M. \& Lo-Coco, F. Arsenic trioxide for management of acute promyelocytic leukemia: current evidence on its role in front-line therapy and recurrent disease. Expert. Opin. Pharm. 13, 1031-1043 (2012).

5. Lo-Coco, F. et al. Retinoic acid and arsenic trioxide for acute promyelocytic leukemia. N. Engl. J. Med. 369, 111-121 (2013).

6. Norsworthy, K. J. \& Altman, J. K. Optimal treatment strategies for high-risk acute promyelocytic leukemia. Curr. Opin. Hematol. 23, 127-136 (2016).

7. Kojima, M. et al. Refractory acute promyelocytic leukemia successfully treated with combination therapy of arsenic trioxide and tamibarotene: a case report. Leuk. Res. Rep. 5, 11-13 (2016)

8. Cai, W. et al. Wanted DEAD/H or alive: helicases winding up in cancers. J. Natl Cancer Inst. 109, 1-15 (2017).

9. Mazurek, A. et al. Acquired dependence of acute myeloid leukemia on the DEAD-box RNA helicase DDX5. Cell Rep. 7, 1887-1899 (2014).

10. Lin, S. et al. DDX5 is a positive regulator of oncogenic NOTCH1 signaling in T cell acute lymphoblastic leukemia. Oncogene 32, 4845-4853 (2013).
} 
11. Misaki, R. et al. Recombinant production and characterization of human antiinfluenza virus monoclonal antibodies identified from hybridomas fused with human lymphocytes. Biologicals 44, 394-402 (2016).

12. Soni, P. et al. Evaluation of the fusion partner cell line SPYMEG for obtaining human monoclonal antibodies against influenza B virus. J. Vet. Med. Sci. 80, 1020-1024 (2018).

13. Chen, $H$. et al. NADPH oxidase-derived reactive oxygen species are involved in the HL-60 cell monocytic differentiation induced by isoliquiritigenin. Molecules 17, 13424-13438 (2012)

14. Callens, C. et al. Targeting iron homeostasis induces cellular differentiation and synergizes with differentiating agents in acute myeloid leukemia. J. Exp. Med. 207, 731-750 (2010)

15. Zhang, Y., Choksi, S., Chen, K., Pobezinskaya, Y. \& Linnoila, I. ROS play a critical role in the differentiation of alternatively activated macrophages and the occurrence of tumor-associated macrophages. Cell Res. 23, 898-914 (2013).

16. Cicconi, L. \& Lo-Coco, F. Current management of newly diagnosed acute promyelocytic leukemia. Ann. Oncol. 27, 1474-1481 (2016).

17. Ling, S. et al. Metformin ameliorates arsenic trioxide hepatotoxicity via inhibiting mitochondrial complex I. Cell Death Dis. 8, e3159 (2017).

18. Stahl, M. \& Tallman, M. S. Differentiation syndrome in acute promyelocytic leukaemia. Br. J. Haematol. 187, 157-162 (2019).

19. Wu, J. et al. The roles and applications of autoantibodies in progression, diagnosis, treatment and prognosis of human malignant tumours. Autoimmun. Rev. 16, 1270-1281 (2017).

20. Igusa, T. et al. Autoantibodies and scleroderma phenotype define subgroups at high-risk and low-risk for cancer. Ann. Rheum. Dis. 77, 1179-1186 (2018).

21. Mao, S., Shen, H. \& Zhang, J. Systemic lupus erythematosus and malignancies risk. J. Cancer Res. Clin. 142, 253-262 (2016).

22. Kantarjian, $\mathrm{H}$. et al. Blinatumomab versus chemotherapy for advanced acute lymphoblastic leukemia. N. Engl. J. Med. 376, 836-847 (2017).

23. Marcus, R. et al. Obinutuzumab for the first-line treatment of follicular lymphoma. N. Engl. J. Med. 377, 1331-1344 (2017).
24. Nyamao, R. M., Wu, J., Yu, L., Xiao, X. \& Zhang, F. M. Roles of DDX5 in the tumorigenesis, proliferation, differentiation, metastasis and pathway regulation of human malignancies. BBA 1871, 85-98 (2019).

25. Ponomartsev, N. V. \& Enukashvily, N. E. The DDX5 protein is involved in cell proliferation and differentiation. Tsitologiia 57, 111-118 (2015).

26. Sha, $M$. et al. Long non-coding RNA MIAT promotes gastric cancer growth and metastasis through regulation of miR-141/DDX5 pathway. J. Exp. Clin. Cancer Res. 37, 58-70 (2018).

27. Wang, Z. D. et al. DDX5 promotes proliferation and tumorigenesis of nonsmall-cell lung cancer cells by activating $\beta$-catenin signaling pathway. Cancer Sci. 106, 1303-1312 (2015).

28. Moeenrezakhanlou, A., Shephard, L., Lam, L. \& Reiner, N. E. Myeloid cell differentiation in response to calcitriol for expression CD11b and CD14 is regulated by myeloid zinc finger-1 protein downstream of phosphatidylinositol 3-kinase. J. Leukoc. Biol. 84, 519-528 (2008).

29. Wang, R., Xia, L., Gabrilove, J., Waxman, S. \& Jing, Y. Sorafenib inhibition of Mcl1 accelerates ATRA-induced apoptosis in differentiation-responsive AML cells. Clin. Cancer Res. 22, 1211-1221 (2016).

30. Amanzadeh, A. et al. New synergistic combinations of differentiation-inducing agents in the treatment of acute promyelocytic leukemia cells. Leuk. Res. $\mathbf{6 8}$ 98-104 (2018).

31. Chen, P. et al. Nuclear localizing sequences promote nuclear translocation and enhance the radiotoxicity of the anti-CD33 monoclonal antibody HuM195 labeled with 111 In in human myeloid leukemia cells. J. Nucl. Med. 47, 827-836 (2006).

32. Duzkale, $\mathrm{H}$. et al. Bone marrow purging studies in acute myelogenous leukemia using the recombinant anti-CD33 immunotoxin HuM195/rGel. Biol. Blood Marrow Transplant. 9, 364-372 (2003).

33. Karsten, C. M. \& Kohl, J. The immunoglobulin, lgG Fc receptor and complement triangle in autoimmune diseases. Immunobiology 217, 1067-1079 (2012).

34. Weisbart, R. H. et al. DNA-dependent targeting of cell nuclei by a lupus autoantibody. Sci. Rep. 5, 1-6 (2015). 